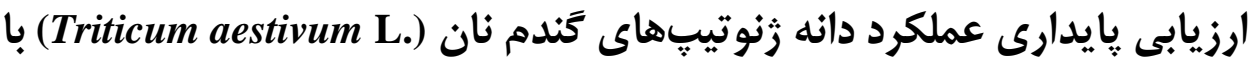

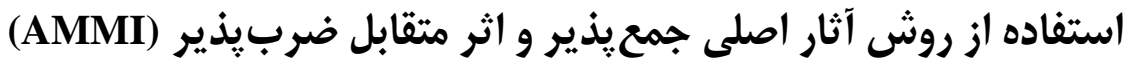

\author{
سعيد عمرانى '، امير محمد ناجى 'و محسن اسماعيلزاده مقدمّ \\ 1- دانشآموخته كارشناسىارشد، گروه زراعت و اصلاح نباتات، دانشخاه شاهد، تهران، ايران، (نويسنده مسوول: s.omrani70@gmail.com)

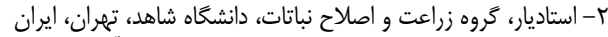

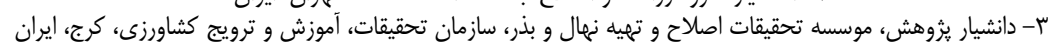 \\ تاريخ دريافت:
}

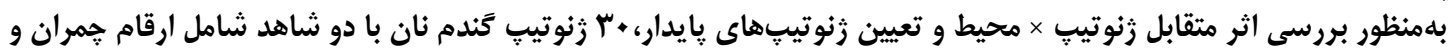

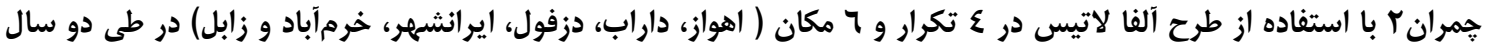

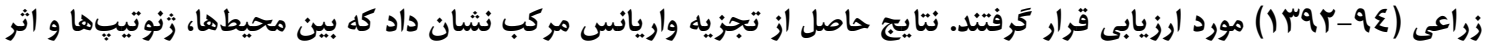

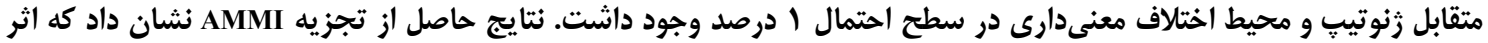

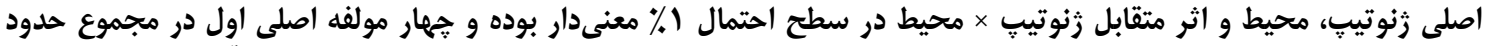

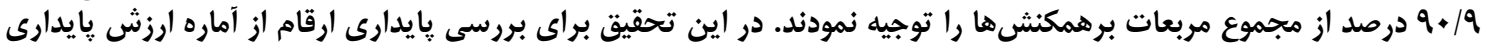
(ASV) AMMI

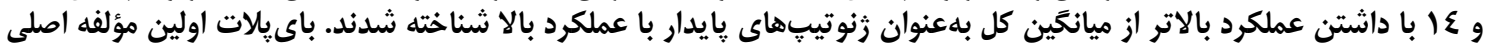

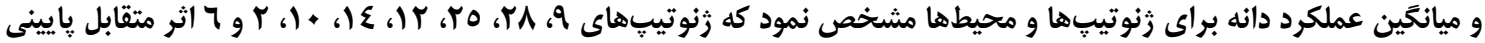

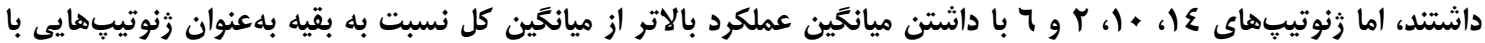

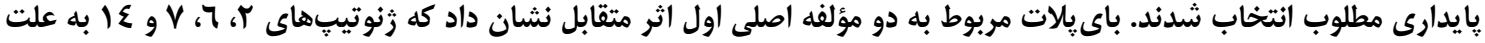

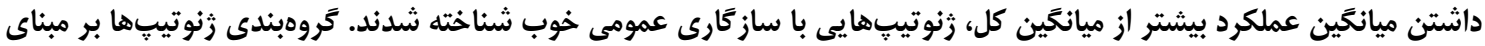

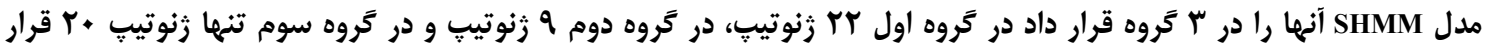

ريك (^)، واريانس بايدارى شوكلا (9) و مدل امى كَج و زوبل

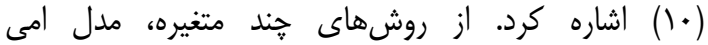

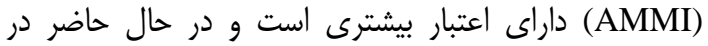

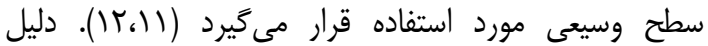

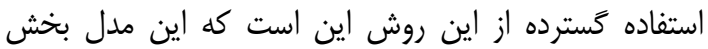

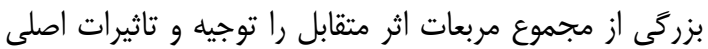

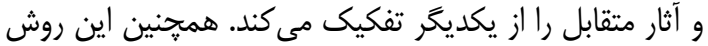

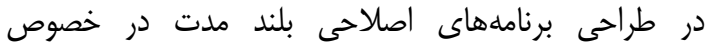

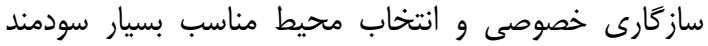

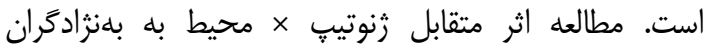

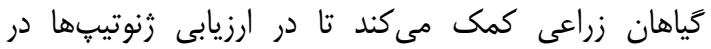

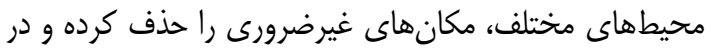

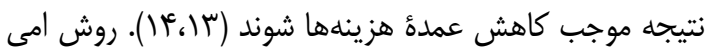

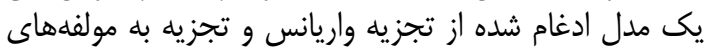

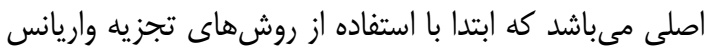

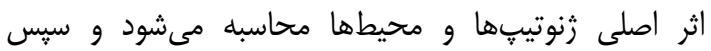

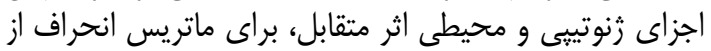

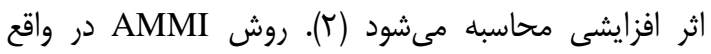

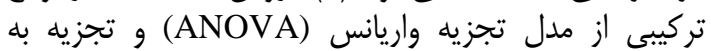

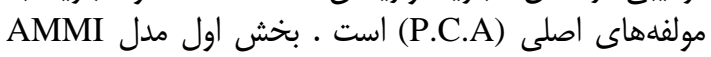

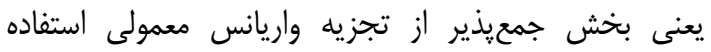

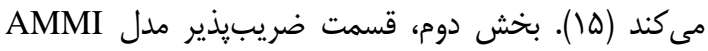

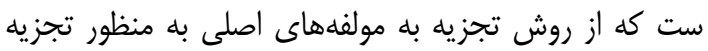
اثر متقابل رنوتيب و محيط به يك تا N مولفه اصلى استفاده
مقدمه

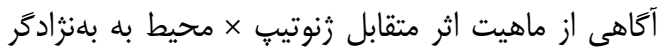

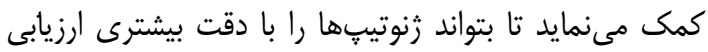

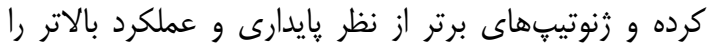

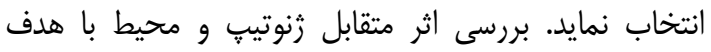

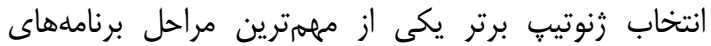

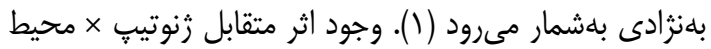

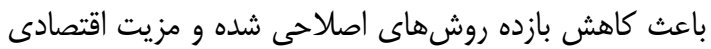

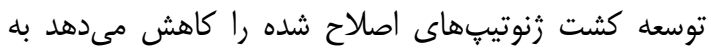

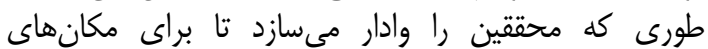

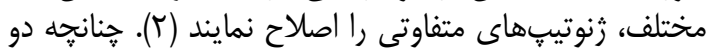

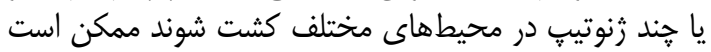

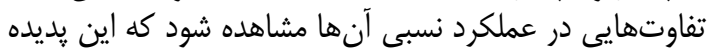

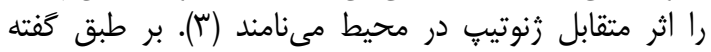

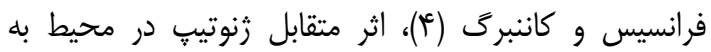

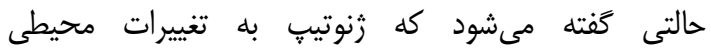

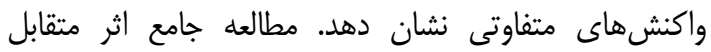

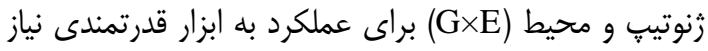

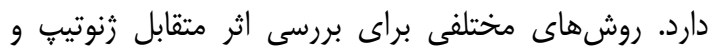

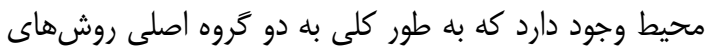

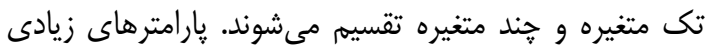

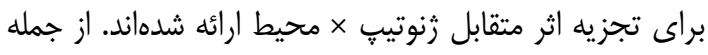

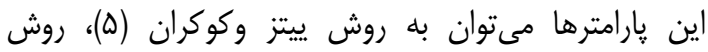

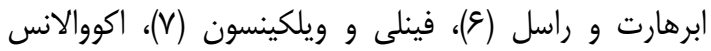


كرفت. در جدول ا، مشخصات جغرافيايى ايستخاههاى

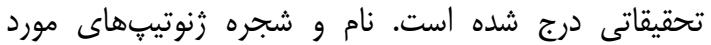

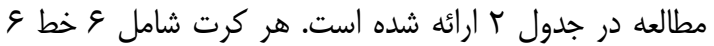

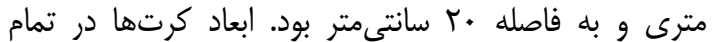

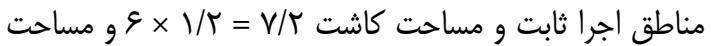

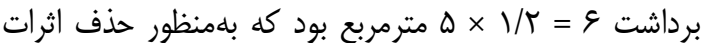

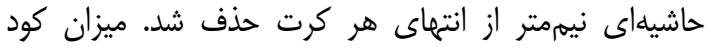

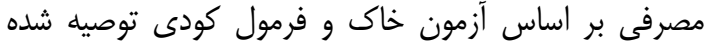

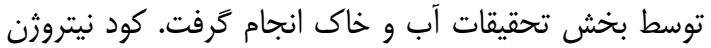

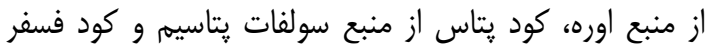

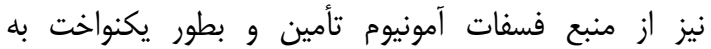

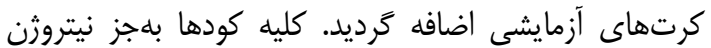

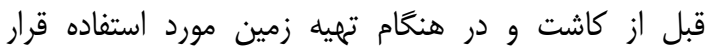

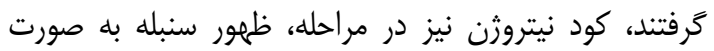

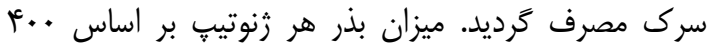

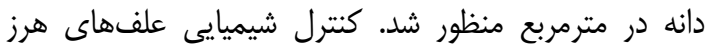

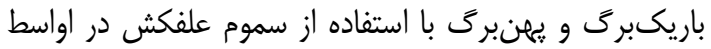

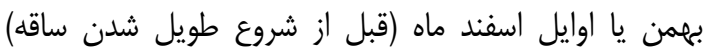

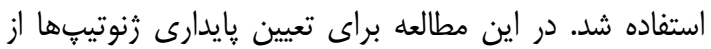

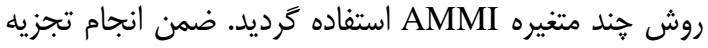

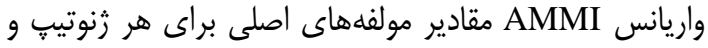

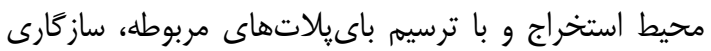

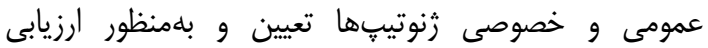

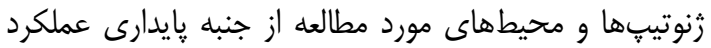

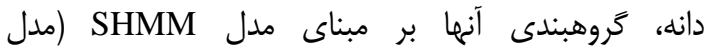

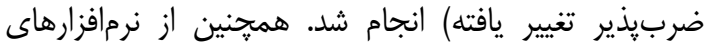
SAS نمودارها استفاده كرديد.
مى كند (ع). در روش SHMM محيطها و زنوتيِها بر يايه

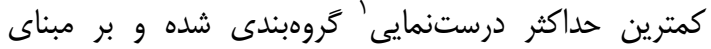

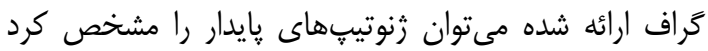

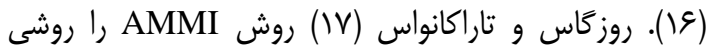

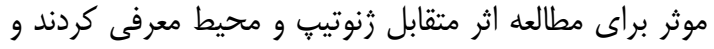

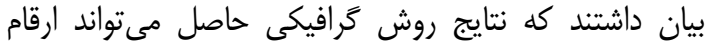

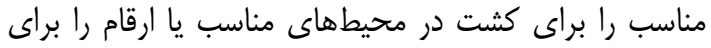

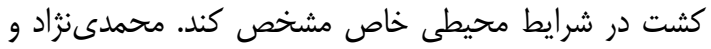

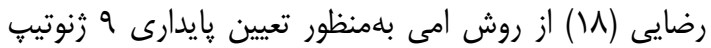

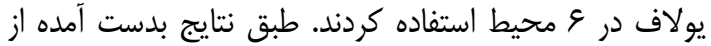

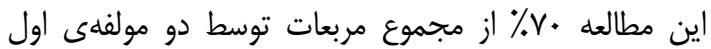

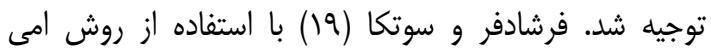

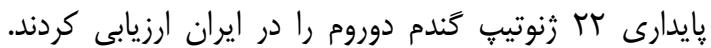
نتايج نشان داد كه

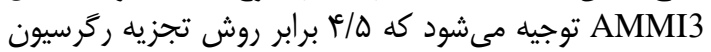

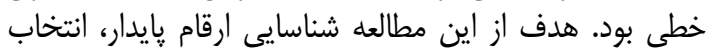

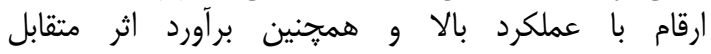

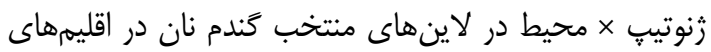

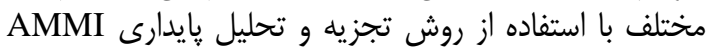
بوده است.

\section{مواد و روشها}

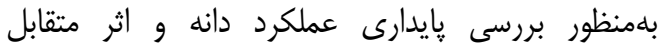

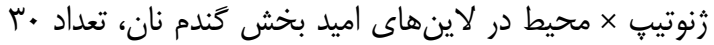

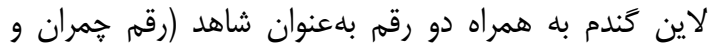

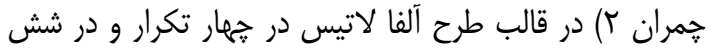

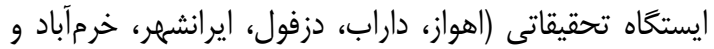

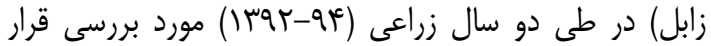

جدول 1-ويزگى هاى جغرافيايى و هواشناسى مكانهاى آزمايشى

Table 1. Meteorological and geographical characteristics of the experimental locations

\begin{tabular}{|c|c|c|c|c|}
\hline مكان & ارتفاع (m) & عرض جغرافيايى & طول جغرافيايى & ميزان بارندگى ساليانه (mm) \\
\hline خرم آباد & $\| f V / A$ & rror.'N & $F \Lambda^{\circ} T D^{\prime} \mathrm{E}$ & 0.9 \\
\hline أهواز & $r / \Delta$ & MIT.'N & $\kappa \mathcal{N}^{\circ} \varphi^{\circ} \cdot \mathrm{E}$ & $r \mid r / k$ \\
\hline دزفول & سאו & rrot.'N & Кペץ.'E & $f \cdot c / s$ \\
\hline زابل & $\uparrow \wedge q / r$ & $\mu^{\circ} \cdot \mathrm{N}$ & SI'T E & 81 \\
\hline داراب & $11 \cdot v$ & $r \Lambda^{\circ} \omega \cdot \mathrm{N}$ & QF०广.'E & $r q T / V$ \\
\hline ايرانشهر & $\Delta 91 / 1$ & $r V^{\circ} \backslash Q^{\prime} \mathrm{N}$ & G. ${ }^{\circ} \cdot{ }^{\circ} \mathrm{E}$ & $1 \cdot \Delta / \Delta$ \\
\hline
\end{tabular}


Table 2. Entry number and pedigree of bread wheat genotypes

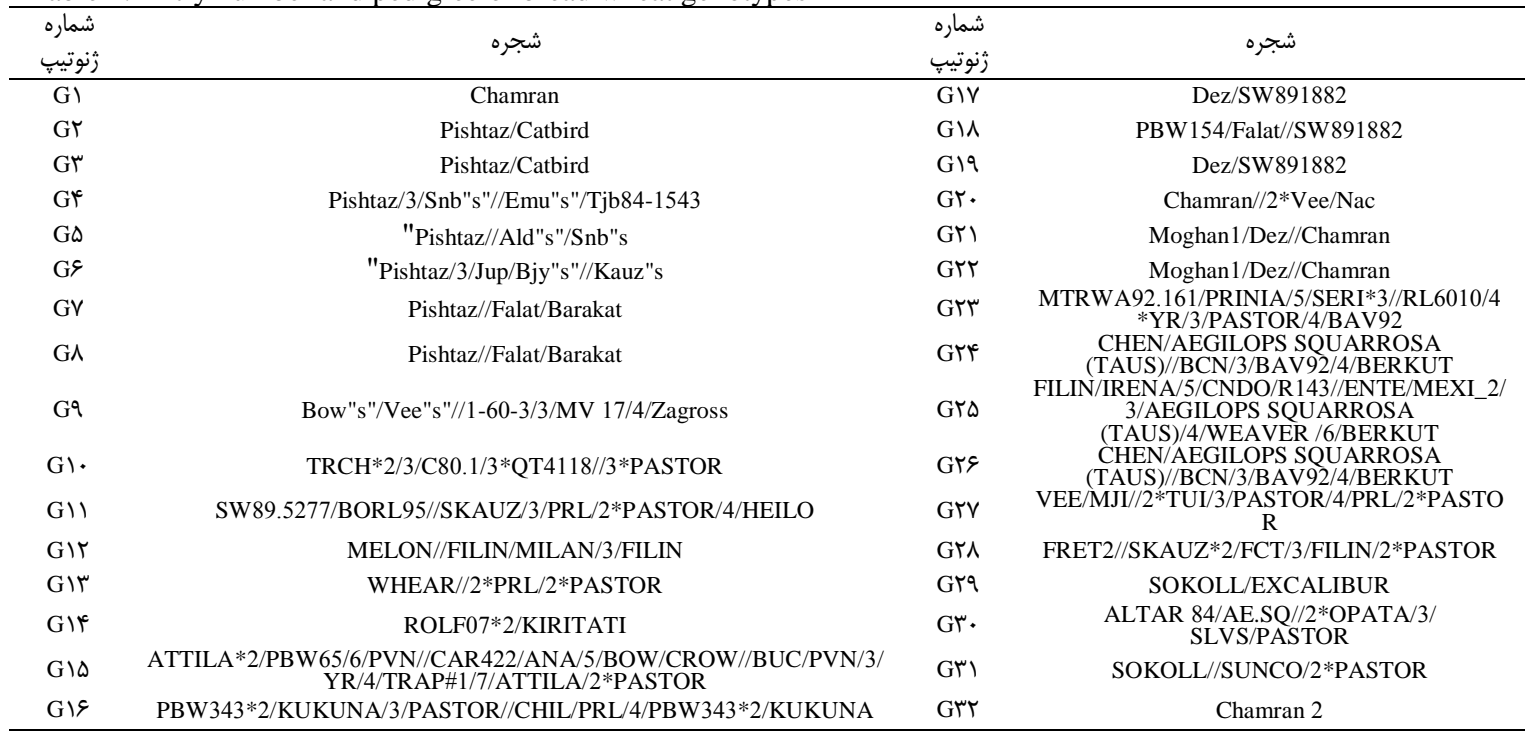

درصد از مجموع مربعات اثر متقابل را به خود اختصاص داد مادند

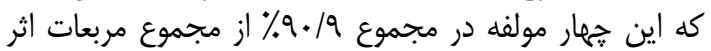

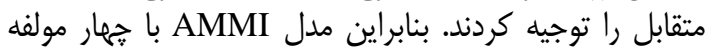

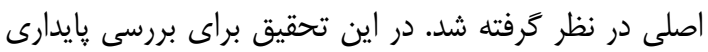

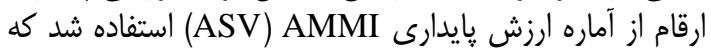

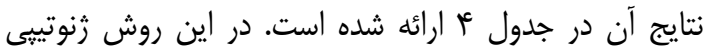

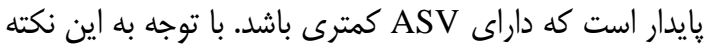

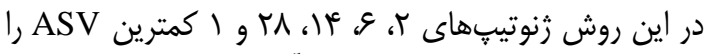

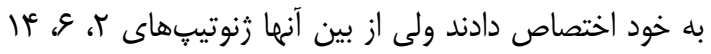

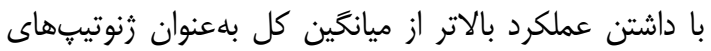

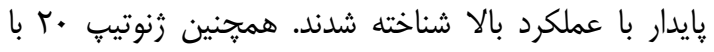

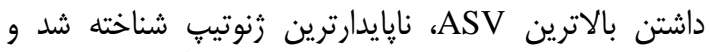

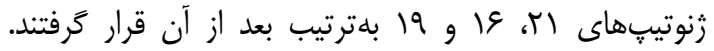

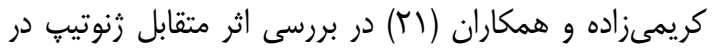
محيط با استفاده از روش

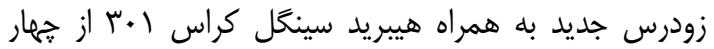

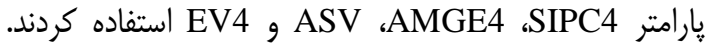

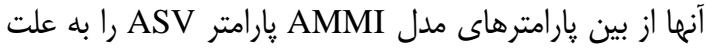

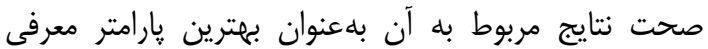
كردند.

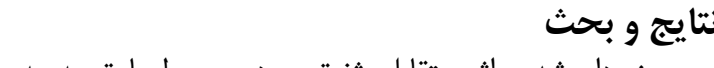

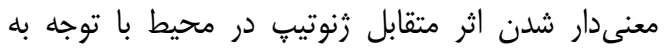

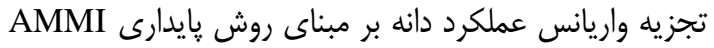

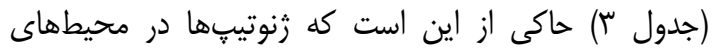

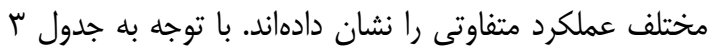

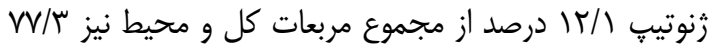

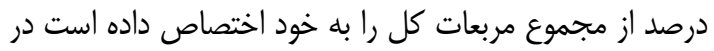

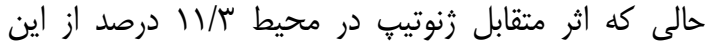

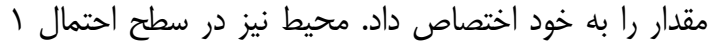

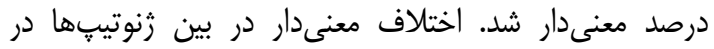

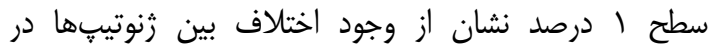

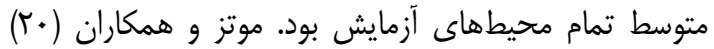

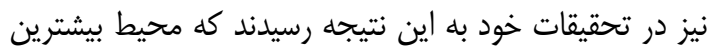

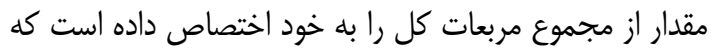

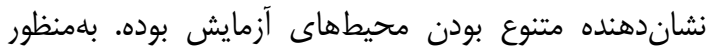

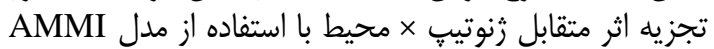

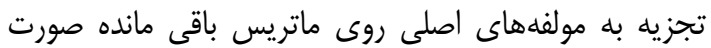

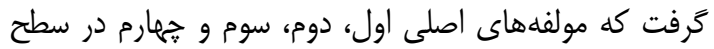
احتمال ا درصد معنىدار شدند. مولفه اصلى اولى اول (IPCA1)

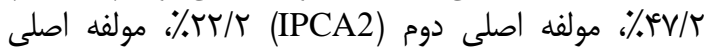

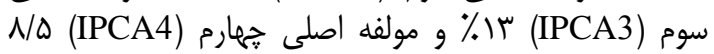




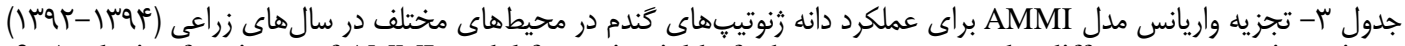
Table 3. Analysis of variance of AMMI model for grain yield of wheat genotypes under different agronomic regimes (2013-2015)

\begin{tabular}{|c|c|c|c|c|}
\hline ميانگين & $\% \mathrm{SS}$ & مجموع & درجه & \multirow{2}{*}{ منابع تغييرات } \\
\hline مربعات & & مربعات (SS) & آزادى & \\
\hline & & $1 \% 94 / \Lambda$ & $V Y V$ & كل \\
\hline$\Delta / \Delta^{* *}$ & & $1 \cdot \Delta s$ & 191 & تيمار \\
\hline $\ln / \mathrm{r}^{* * *}$ & $V V / r$ & $11 \xi / 8$ & $\Delta$ & محيط \\
\hline$\cdot / V^{* *}$ & $11 / r$ & $119 / 1$ & $1 \Delta \Delta$ & اثر متقابل رنوتيب × محيط \\
\hline$\cdot 10^{* *}$ & $1 \%$ & $10 / 9$ & 山 & $\mathrm{IPCA}_{3}$ \\
\hline$\cdot / \mu^{* * *}$ & $N / \Delta$ & $1 \cdot / 4$ & rq & $\mathrm{IPCA}_{4}$ \\
\hline . $/ \mu^{\text {ns }}$ & $\Delta / 1$ & $s / r$ & DT & باقيمانده (نويز) \\
\hline & & 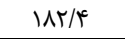 & AVE & خطاى ادغام شده \\
\hline
\end{tabular}

جدول זٔ- ميانگين عملكرد دانه، ارزش يايدارى و مقادير مولفه هاى اصلى زنوتيِهاى گندم نان Table 4. Mean grain yield, stability value and Principle component of bread wheat genotypes

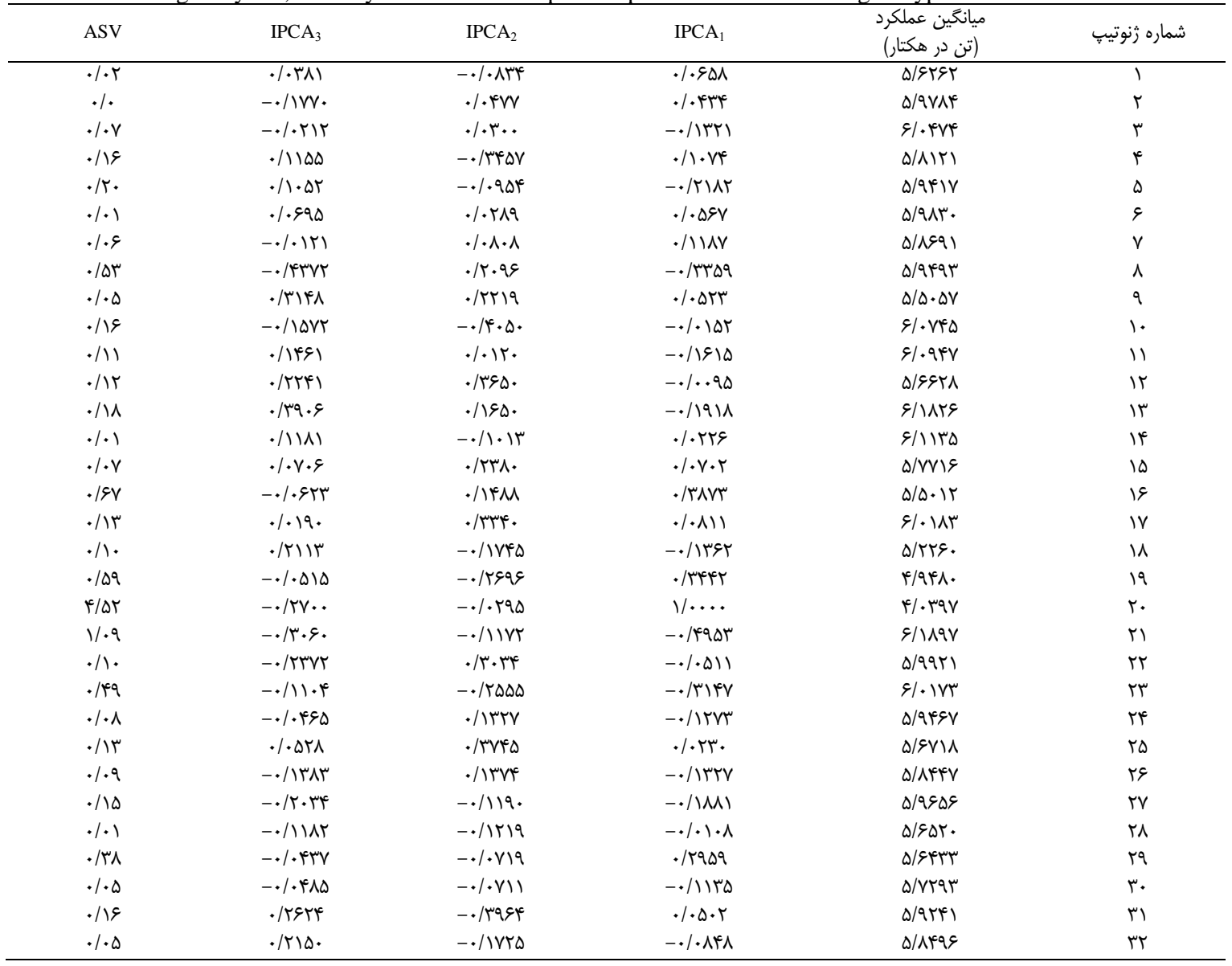

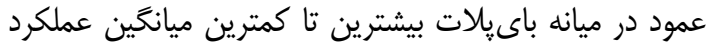

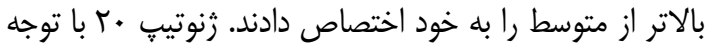

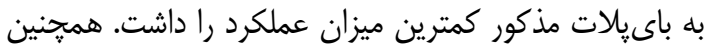

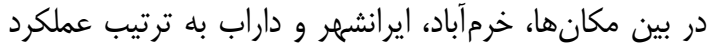

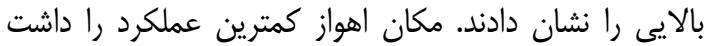

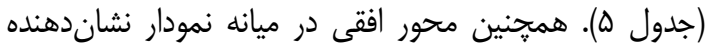
(AMMI= 0) IPCA1=0

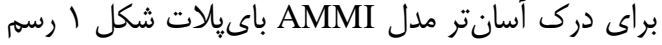

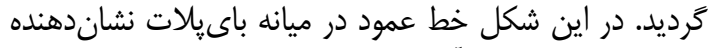

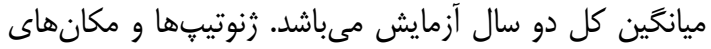

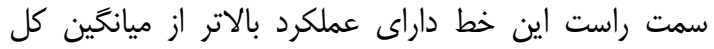

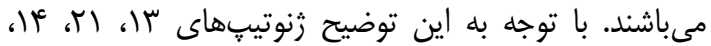

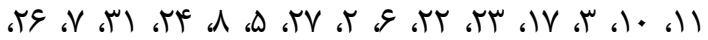
זس، أ و ها به ترتيب با قرار گرفتن در سمت راست خط 


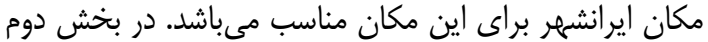

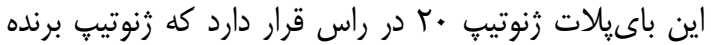

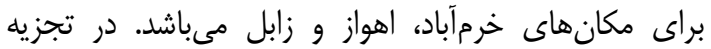

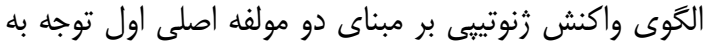

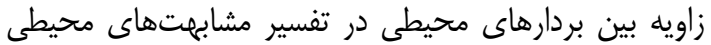

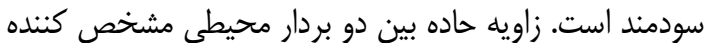

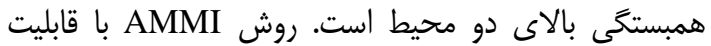

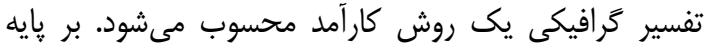

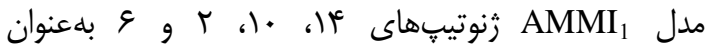

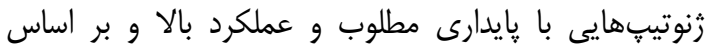
مدل رنو

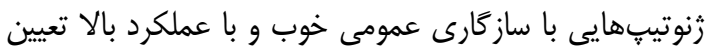

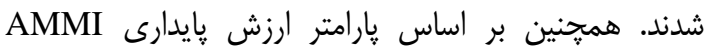

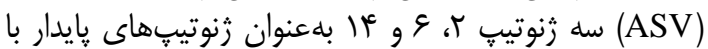

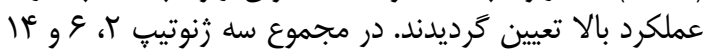

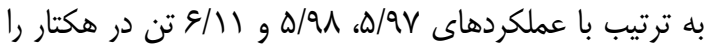

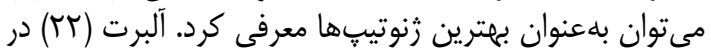

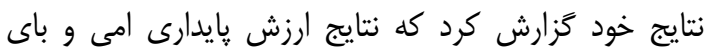

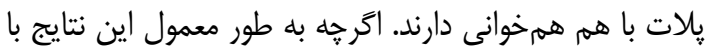

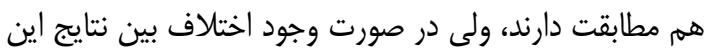

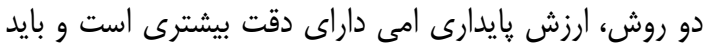

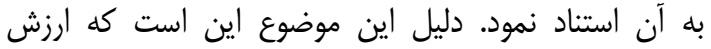

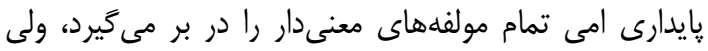

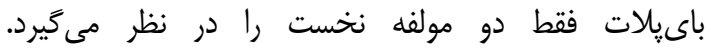

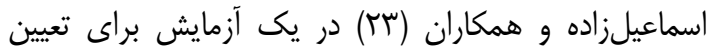

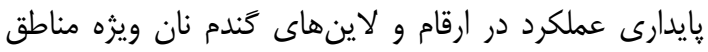

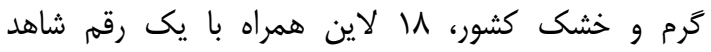

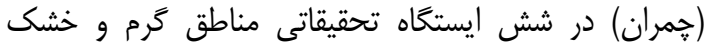

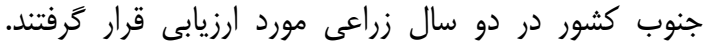

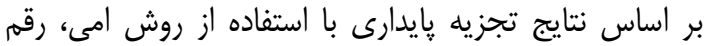
Weebili

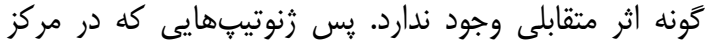

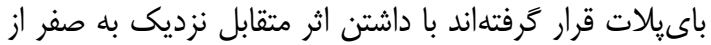

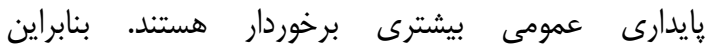

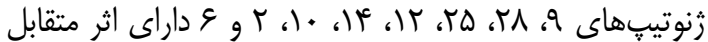

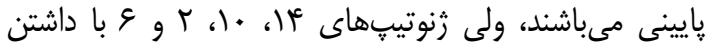

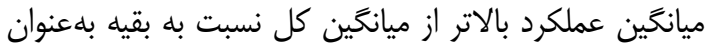

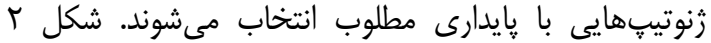

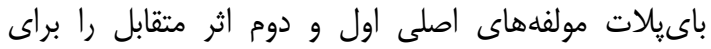

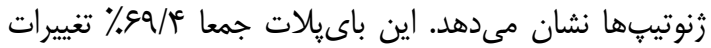

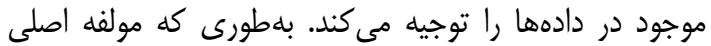

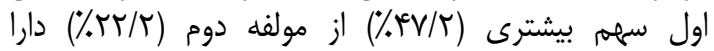

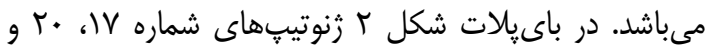

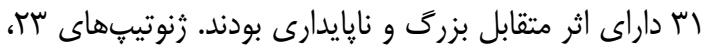

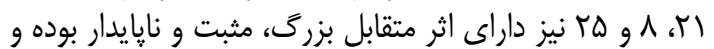

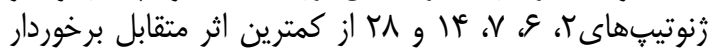

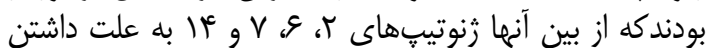

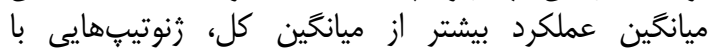

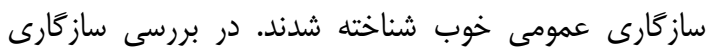

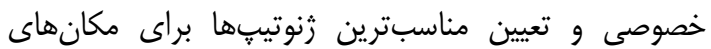

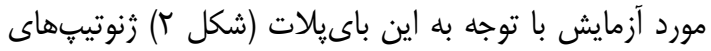

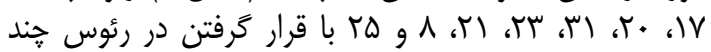

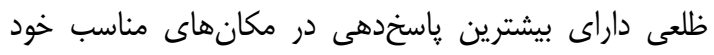

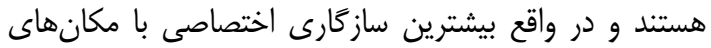

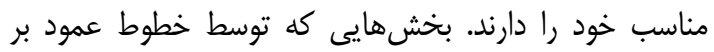

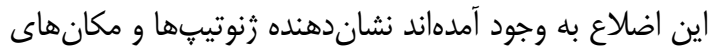

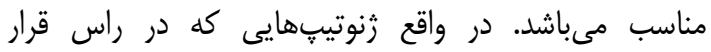

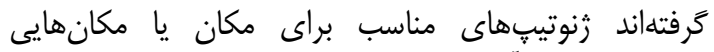

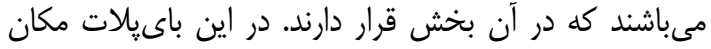

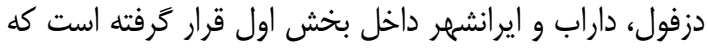

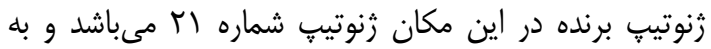

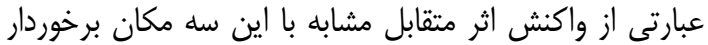

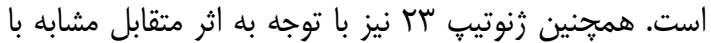

جدول ه- ميانگين عملكرد دانه و مقادير مولفههاى اصلى براى مكانهاى ا تا \& Table 5. The mean grain yield and the Principle component for sites 1 to 6

\begin{tabular}{|c|c|c|c|c|c|}
\hline نام مكان & كد مكان & ميانگين & IPCA1 & IPCA2 & IPCA3 \\
\hline اهواز & S1 & $F / 1 f q \Delta$ & - /TEIA & $\cdot 1 \cdot 1 \cdot 1$ &.$- /$ Irvq \\
\hline داراب & S2 & o/9/VI & זמזא/.- & . Iffeg & $\cdot / r \cdot v q$ \\
\hline دزفول & S3 & $\Delta / V F V D$ & $-\cdot / \mathrm{F} \Delta \mathrm{V}$ & . /reqR & . IETr. \\
\hline ايرانشهر & S4 & \&/Т०१९ & $-\cdot /$ VTS & $-\cdot / D I D T$ & $-\cdot / 9 \cdot 11$ \\
\hline خرم آباد & S5 & V/DVYF & ./MNV & $-\cdot /$ VIVe & 1 \\
\hline زابل & S6 & r/99v9 & . DTHT & - /VASF & 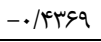 \\
\hline
\end{tabular}




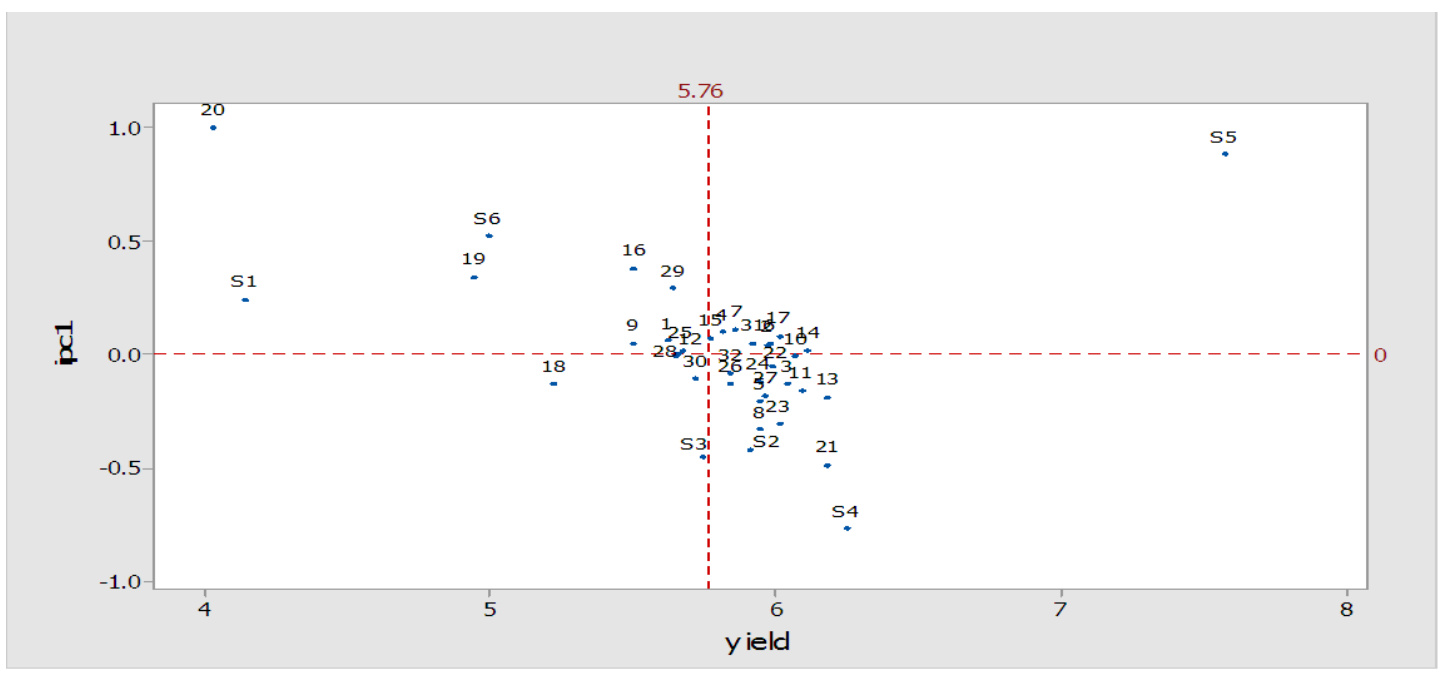

شكل ا- باىيلات ميانكين رنوتيهها و اولين مولفه اصلى براى زنوتيهها و مكانهاى مورد بررسى (S1 تا S6 به ترتيب اهواز، داراب، دزفول، ايرانشهر، خرم آباد و زابل مىباشندان ماندان

Figure 1. Mean yield Biplot of genotypes for 6 locations studies with first their principle component

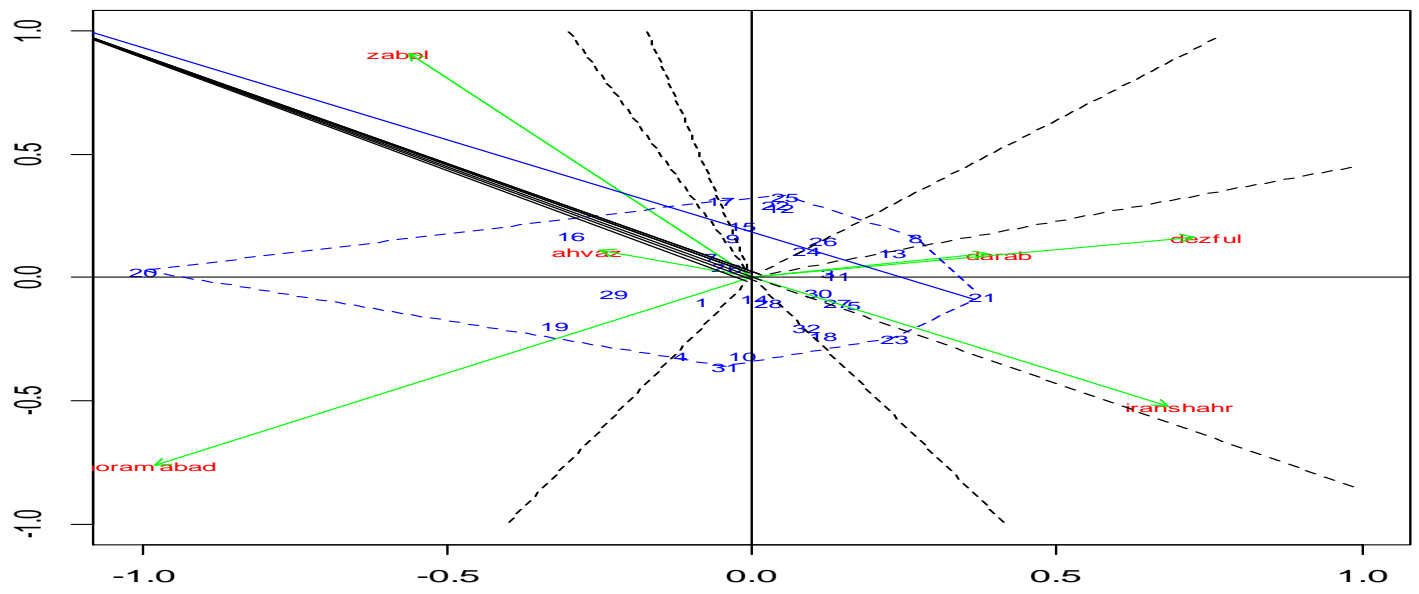

شكل r- باى يلات دو مولفه اصلى اول و دوم براى زنوتيیها و مكانهاى مختلف

Figure 2. Bipolt of the first and second principle components for genotypes and different locations

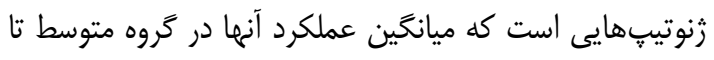

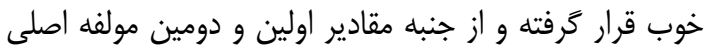

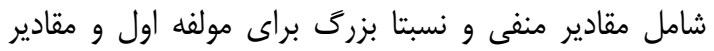

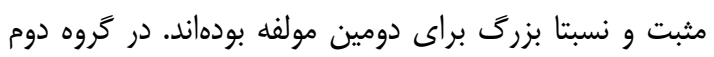

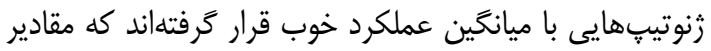

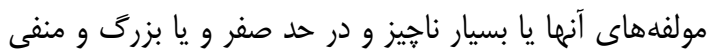

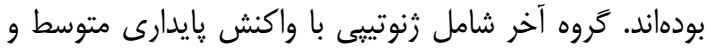
ميانگين عملكرد ضعيف قرار داشت.
بلهمنظور ارزيابى زنوتيڤهاى مورد مطالعه از جنبه پايدارى

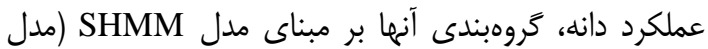

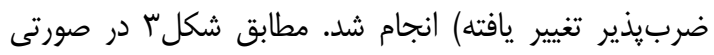
كه كلاستر با فرمول

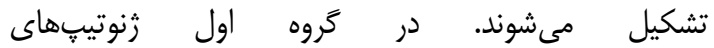

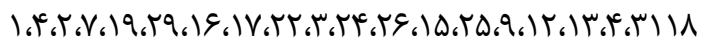

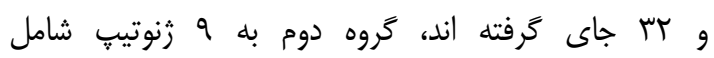

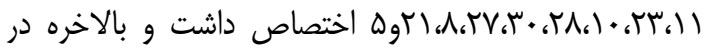
كروه سوم فقط زنوتيت .ب قرار داشت. گروه اول شامل 


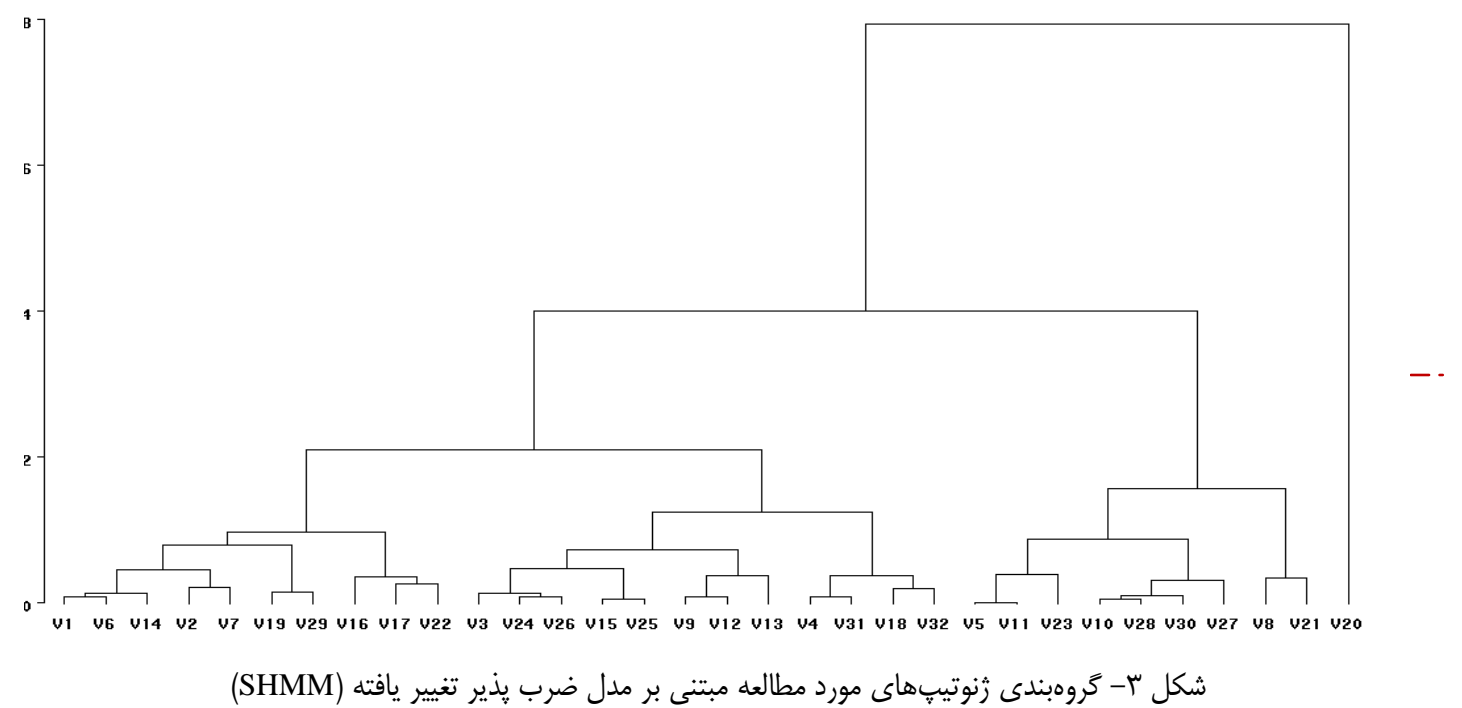

Figure 3. Classification of studied genotypes based on Shifted multiplicative models cluster analysis (SHMM)

1. Kaiser, D.E., J.J. Wiersma and J.A. Anderson. 2014. Genotype and environment variation in elemental composition of spring wheat flag leaves. Agronomy Journal, 106: 324- 336.

2. Crossa, J., H.G. Gauch and R.W. Zobel. 1990. Additive main effects and multiplicative interaction analysis of two international maiz cultivar trials. Crop Science, 30: 493-500.

3. Peterson, C.J., M. Moffatt and J.R. Erickson. 1997. Yield stability of hybrid vs. pure line hard winter wheats in regional performance trials. Crop Science, 37: 116- 120.

4. Francis, T.R. and L.W. Kannenberg. 1978. Yield stability sudies in short-season Maize: 1.A descriptive method for grouping genotypes. Canadian Journal of Plant Science, 58: 1029-1034.

5. Yates, F. and W.G. Cochran. 1938. The analysis of groups of experiments. Journal of Agricultural Science, 28: 556-580.

6. Eberhart, S.A. and W.A. Russell. 1966. Stability parameters for comparing varieties. Crop Science, 6: 3640.

7. Finlay, K.W. and G.N. Wilkinson. 1963. The analysis of adaptation in a plant breeding program. Australian Journal of Agricultural Research, 14: 742-754.

8. Wricke, G. 1962. Uber eine methode zur refassung der okologischen streubretite in feldversuchen, Flazenzuecht, 47: 92-96.

9. Shukla, G.K. 1972. Some statistical aspects of partitioning genotype-environmental components of variability. Heredity, 29: 237-245.

10. Gauch, H.G. and R.W. Zobel. 1988. Predictive and postdictive success of statistical analyses of yield trials. Theoretical and Applied Genetics, 76: 1-10.

11. Annicchiarico, P., L. Russi, E. Piano and F. Veronesi. 2006. Cultivar adaptation across Italian locations in four turforass species. Crop Science, 46: 264-272.

12. Moreno-Gonzalez, J., J. Crossa and P.L. Cornelius. 2004. Genotype x environment interaction in multienvironment trials using shrinkage factors for AMMI models. Euphytica, 137: 119-127.

13. Yan, W., M.S. Kang, B. Ma, S. Woods and P.L. Cornelius. 2007. GGE biplot vs. AMMI analysis of genotype-by-environment data. Crop Science, 47: 643-655.

14. Basford, K.E. and M. Cooper. 1998. Genotype by environment interactions andsome considerations of their implication for wheat breeding in Australia. Australian Journal of Agricultural Research, 49: 154-175.

15. Gauch, H.G. 1992. Statistical Analysis of Regional Trials. AMMI Analysis of Factorial Designs. Elsevier Pub. Amsterdam, the Netherlands, 51: 242-244.

16. Gauch, H.G. and R.W. Zobel. 1997. Identifying mega-environments and targeting genotypes. Crop Science, 37: 311-326.

17. Tarakanovas, P. and V. Ruzgas. 2006. Additive main effect and multiplicative interaction analysis of grain yield of wheat varieties in Lithuania. Agronomy Research, 4: 91-98.

18. Mohammadinejad, G. and A.M. Rezai. 2005. Analysis of genotype $\times$ environment interaction and study of oat (Avena sativa L.) genotypes pattern. Journal of Science and Technology of Agriculture and Natural Resources, 9:77-89 (In Persian).

19. Farshadfar, E. and J. Sutka. 2006. Biplot analysis of genotype-environment interaction in durum wheat using the AMMI model. Acta Agronomica Hungarica, 54: 459-467.

20. Motzo, R., F. Guinta and M. deidda. 1962. Factors affecting thegenotypex environment interaction in spring triticale grown in Mediterranean environment. Euphytica, 121: 317-324.

21. Karimi Zadeh, R., H. Dehghani and Z. Dehghanpour. 2008. Use of AMMI method for estimating genotype $x$ environment interaction in early maturing corn hybrids. Seed Plant Improve, 23: 531-546 (In Persian).

22. Albert, J. A. 2004. Comparison of Statistical Methods to Describe Genotype x Environment Interaction and Yield Stability in Multi-Location Maize Trials. M.Sc. Thesis, University of the Free State, Bloemfontein.

23. Esmailzadeh, M., M. Moghaddam, M. Zakizadeh, H. Akbari-Moghaddam, M. Abedini-Esfahlani, M. Sayahfar, A.R. Nikzad, S. Tabib-Ghafari, M. Lotfi and G.A. Ayene. 2011. Genotype $\times$ environment interaction and stability of grain yield of bread wheat genotypes in dry and warm areas of Iran. Seed Plant Improve, 27: 257-273 (In Persian). 


\title{
Evaluation of Yield Stability of Bread wheat (Triticum aestivum L.) Genotypes using Additive Main Effects and Multiplicative Interaction (AMMI)
}

\author{
Saeed Omrani ${ }^{1}$, Amir Mohammad Naji ${ }^{2}$ and Mohsen Esmaeil Zadeh Moghadam ${ }^{3}$ \\ 1- Graduated M.Sc. Student, Department of Plant Breeding, Shahed University, Tehran, Iran \\ (Corresponding Author: s.omrani70@gmail.com) \\ 2- Assistant Professor, Department of Plant Breeding, Shahed University, Tehran, Iran \\ 3- Associated Professor, Seed and Plant Improvement Institute, Agricultural Research Education and Extension \\ Organization (AREEO), Karaj, Iran \\ Received: June 5, $2016 \quad$ Accepted: September 21, 2016
}

\begin{abstract}
To evaluate the genotype $\times$ environment interaction and determine the stable genotypes of wheat, 30 genotypes of bread wheat along with two controls namely Chamran and Chamran 2 were studied in 6 locations (Ahwaz, Darab, Dezful, Iranshahr, Khorramabad, Zabul) and two years (from 2013 to 2015), in each using an alpha lattice design with 4 replications. The results obtained from AMMI analysis demonstrated that the main effects of genotype, environment, genotype $\times$ environment interaction and the first four principal components were highly significant. The first four principal components justified around $90.9 \%$ of the sum of squares of the interactions. By using the stability of the figures of the statistics lasting value AMMI (ASV), genotypes 2, 6, 14, 28 had the lowest (ASV) values. Genotypes 2, 6 and 14 with higher yields than the overall mean were identified as high yielding genotypes with stable performance. Drawing the biplot of the first principal component and the average yield for genotypes and environments suggested that genotypes $9,28,25,12,14,10,2$ and 6 had low interactions, but genotypes 14, 10, 2 and 6 with higher than average yields and desired stability were selected. Biplot of the first two principal components showed that the interaction between genotypes 2,6 , 7 and 14 due to higher grain yield than the average of the total, were identified genotypes with good compatibility. Genotype grouping them into three groups based on the model SHMM placed in the first group of 22 genotypes, genotypes 9 in the second group and the third group was the only genotype 20 .
\end{abstract}

Keywords: AMMI, Compatibility, Genotype $\times$ Environment interaction, Wheat, Yield stability 